\title{
Mindfulness, burnout, and effects on performance evaluations in internal medicine residents
}

This article was published in the following Dove Press journal:

Advances in Medical Education and Practice

16 August 2017

Number of times this article has been viewed

\author{
Sarah E Braun' \\ Stephen M Auerbach' \\ Bruce Rybarczyk' \\ Bennett Lee ${ }^{2}$ \\ Stephanie Call ${ }^{2}$ \\ 'Department of Psychology, School \\ of Humanities and Sciences, \\ Virginia Commonwealth University, \\ Richmond,VA, USA; ${ }^{2}$ Department of \\ Internal Medicine, Division of General \\ Medicine, Virginia Commonwealth \\ University, Richmond, VA, USA
}

Correspondence: Sarah E Braun Department of Psychology, School of Humanities and Sciences, Virginia Commonwealth University, 806 West Franklin Street, Richmond, VA 23284, USA

Email braunse2@vcu.edu
Purpose: Burnout has been documented at high levels in medical residents with negative effects on performance. Some dispositional qualities, like mindfulness, may protect against burnout. The purpose of the present study was to assess burnout prevalence among internal medicine residents at a single institution, examine the relationship between mindfulness and burnout, and provide preliminary findings on the relation between burnout and performance evaluations in internal medicine residents.

Methods: Residents $(n=38)$ completed validated measures of burnout at three time points separated by 2 months and a validated measure of dispositional mindfulness at baseline. Program director end-of-year performance evaluations were also obtained on 22 milestones used to evaluate internal medicine resident performance; notably, these milestones have not yet been validated for research purposes; therefore, the investigation here is exploratory.

Results: Overall, $71.1 \%(n=27)$ of the residents met criteria for burnout during the study. Lower scores on the "acting with awareness" facet of dispositional mindfulness significantly predicted meeting burnout criteria $\chi^{2}(5)=11.88, p=0.04$. Lastly, meeting burnout criteria significantly predicted performance on three of the performance milestones, with positive effects on milestones from the "system-based practices" and "professionalism" domains and negative effects on a milestone from the "patient care" domain.

Conclusion: Burnout rates were high in this sample of internal medicine residents and rates were consistent with other reports of burnout during medical residency. Dispositional mindfulness was supported as a protective factor against burnout. Importantly, results from the exploratory investigation of the relationship between burnout and resident evaluations suggested that burnout may improve performance on some domains of resident evaluations while compromising performance on other domains. Implications and directions for future research are discussed.

Keywords: burnout, mindfulness, internal medicine residents, performance evaluations

\section{Introduction}

Burnout is defined as a state of work-related stress which causes health care professionals to experience emotional exhaustion, depersonalization, and decreased sense of personal accomplishment., ${ }^{1,2}$ High levels of burnout have been documented in physicians and medical residents ${ }^{3-5}$ with costly effects on resident quality of life, patient safety, and the health care system as a whole. ${ }^{6-14}$ Burnout in internal medicine physicians is often reported at higher levels than in other medical specialties. ${ }^{3,5}$ However, the reason for this difference is unclear and may be due to specific work duties or may represent factors unrelated to resident speciality. ${ }^{3,5}$ It is important to advance our understanding of the negative correlates of burnout during medical training to 
inform appropriate interventions for decreasing burnout and its negative sequelae. By focusing on internal medicine residents for whom burnout is reported at high levels, the information gained may inform future research in medical residents across specialties.

Mindfulness has been defined as a state of nonjudgmental awareness in the present moment. ${ }^{15}$ Mindfulness-based interventions may be especially beneficial for decreasing burnout in health care professionals ${ }^{16,17}$ by enhancing attentional capacities under stress and resiliency to stress. ${ }^{18,19}$ Dispositional mindfulness, or the tendency to be mindful in one's daily life, has been shown to increase following mindfulness training, ${ }^{20}$ but also represents an individual's tendency to be mindful unrelated to formal training. ${ }^{21,22}$ Dispositional mindfulness is measured using validated self-report questionnaires and has been positively associated with adaptive health outcomes. $^{21,22}$ However, few studies have investigated the relationship between dispositional mindfulness and burnout in medical residents. ${ }^{23}$ An investigation of this relationship could inform residency program directors on intervention development and help to identify resiliency factors for the prevention of resident burnout.

Across disciplines, occupational stress has been shown to negatively affect cognitive functioning and work-related performance. ${ }^{24-26}$ However, research findings on the relationship between burnout and performance in medical residents have been inconsistent. Burnout tends to correlate negatively with perceived medical errors ${ }^{8,9,12,14}$ and patient satisfaction. ${ }^{6}$ Conversely, when work performance is measured using chart audits ${ }^{27}$ or observed medical errors, ${ }^{28}$ no relation is found between burnout and performance. Others have found positive associations between burnout and poorer examination scores in medical residents. ${ }^{13}$

Notably, burnout is consistently related to anxiety, increased risk for cardiovascular disease, poor adrenal functioning, and suicide. ${ }^{29,30}$ Therefore, it seems likely that burnout negatively affects performance, and the lack of consistent association found between burnout and objective measures of performance in medical residents may be due to issues of operationalizing performance. There may be particular aspects of resident performance that are negatively influenced by the presence of high burnout, specifically patient care or performance on more cognitively demanding tasks. The Internal Medicine resident milestones developed by the Accreditation Council for Graduate Medical Education (ACGME) may give important nuanced understanding of how burnout affects different educational competencies, yet to our knowledge, no research has investigated this relationship. The Internal Medicine resident milestones developed by the ACGME represent a shift toward competency-based education in residency training. ${ }^{31,32}$ These milestones are used to guide evaluation of residents and were developed by a taskforce with expertise in the areas of internal medicine, program evaluation, and graduate medical education. ${ }^{31}$ They are widely used and provide national standardization for graduate training in internal medicine. However, no research has explored their relationship to burnout. Therefore, the present study sought to address this gap in the literature to better understand how burnout may affect internal medicine residents on relevant aspects of their performance.

Here findings are reported on burnout prevalence at a single institution throughout half a residency year, the relationship between dispositional mindfulness (as measured by a self-report scale) and burnout, and on an analysis of the relationship between burnout and Internal Medicine resident milestones developed by the ACGME. It was hypothesized that higher levels of dispositional mindfulness in residents would decrease likelihood of meeting criteria for burnout. Given the conflicting findings relating resident burnout to performance, and the exploratory nature of the final aim, there were no hypotheses made regarding the relationship between burnout and Internal Medicine resident milestones.

\section{Methods}

\section{Participants/procedures}

All internal medicine residents at Virginia Commonwealth University (VCU) were eligible to participate. Residents who agreed to participate provided written informed consent. Ethical approval was granted from the VCU Institutional Review Board (IRB), 7/10/2014-6/30/2015, IRB number HM20001840. Burnout was measured three times separated by 2.5 months beginning with the start of a residency year (July, October, and December). Data on dispositional mindfulness and demographic variables were obtained at baseline. Internal Medicine resident milestones were obtained at study end in December 2014.

\section{Measures}

\section{Burnout}

The Maslach Burnout Inventory (MBI) measures burnout in three domains: emotional exhaustion, depersonalization, and lack of personal accomplishment., ${ }^{1,2}$ The MBI is a 22-item measure asking respondents to rate the frequency of several feelings and experiences on a 7-point Likert scale ranging 
from "never" to "daily". This instrument is the gold standard for measuring burnout in medical professionals and is well validated in physician and resident populations. ${ }^{33}$ Scores of 27 or higher on the emotional exhaustion subscale or scores of 10 or higher on the depersonalization subscale are considered criteria for meeting burnout. ${ }^{1,7,12,34}$ Consistent with previous literature, low scores on the personal accomplishment subscale are not considered burnout, because this may measure a distinct dimension. ${ }^{1}$ The subscales of emotional exhaustion and depersonalization demonstrated adequate to good internal consistency $(\alpha=0.85-0.91$ and $0.77-0.84$, respectively).

\section{Mindfulness}

The Five Facet Mindfulness Questionnaire Short Form (FFMQ-sf) is a 24-item measure, derived from the 39-item FFMQ, which assesses dispositional mindfulness on a fivelevel Likert scale. ${ }^{35}$ The FFMQ-sf captures the tendency to be mindful in one's life using five distinct subscales, "observe", "describe", "acting with awareness", "nonjudging of inner experience", and "non-reactivity to inner experience". Higher scores indicate higher levels of mindfulness. The FFMQ-sf is well validated. ${ }^{35}$ The five facets demonstrated acceptable to excellent internal consistency $(\alpha=0.73-0.91)$.

\section{Stress and depression}

The Depression Anxiety Stress Scales short form (DASS $21)^{36}$ was used to measure stress and depression at baseline as a potential covariate. Only the depression and stress subscales (14 items) were administered. Participants were asked to rate the degree to which statements applied to them over the last week on a 4-point Likert scale ranging from "did not apply to me at all" to "applied to me very much, or most of the time". The DASS 21 is well validated and well normed in non-clinical populations. ${ }^{37}$ The subscales demonstrated adequate to good internal consistency, alphas ranged from 0.78 to 0.81 .

\section{Internal Medicine resident milestones}

For each resident, an aggregate of evaluations from attending physicians and faculty was discussed during a three-person committee meeting made up of department chiefs providing performance scores for each resident on 22 milestones developed by the ACGME, each of which falls under one of the six competencies: "patient care", "medical knowledge", "practice-based learning and improvement", "interpersonal and communication skills"," professionalism", and "systemsbased practice". All milestones were rated on a 5-point scale ranging from "critical deficiencies" to "aspirational" based on nation-wide standards developed by the ACGME. ${ }^{31}$ It was expected that first, second, and third year residents would vary in their milestone ratings as their performance should improve as they advance in years in training. Therefore, performance on each milestone was compared with the residency class to which each participant belonged, to determine whether it fell below the class average or at or above the class average, making performance on each milestone a dichotomous variable.

\section{Statistical plan}

A hierarchical logistic regression was conducted to test the hypothesis that dispositional mindfulness would predict burnout criteria. Chi-square tests were conducted to evaluate whether burnout was associated with performance on the 22 Internal Medicine resident milestones. Data were analyzed using SPSS version 22 (IBM Corporation, Armonk, NY, USA). All data met assumptions for statistical analyses. Importantly, for the hierarchical logistic regression, assumptions of normality were tested via skewness and kurtosis statistics $<1.5$, bivariate and multivariate outliers using Mahalanobis distance, and homoscedasticity of error variance by examining the residual plot. All assumptions were met. Finally, the Hosmer and Lemeshow test was used to determine goodness-of-fit.

\section{Results}

Of the 129 residents in the internal medicine residency program, 38 participated in the study (30\% response rate). One participant did not provide a valid identifier, therefore performance evaluations were collected for 37 residents. Participants were mostly men $(n=30,79 \%)$ and the average age was less than 30 years $(M=28.59, \mathrm{SD}=2.69)$. Half of the sample was White $(\mathrm{n}=19), 26.3 \%(\mathrm{n}=10)$ was Asian, $2.6 \%$ $(\mathrm{n}=1)$ Hispanic, $5.3 \%(\mathrm{n}=2)$ identified as other, and $15.8 \%$ $(n=6)$ chose not to disclose. Most of the participants were interns $(n=20,52.6 \%)$ or third year residents $(n=16,42.1 \%)$, with second year residents underrepresented $(n=2,5.3 \%)$.

\section{Prevalence of burnout}

Of the 38 residents surveyed, 27 (71.1\%) met criteria for burnout at least once during the course of the study. At any of the three assessments, $12-19(31.6 \%-50.0 \%)$ residents met criteria for burnout. Average burnout ranged from moderate to severe (Table 1). These prevalence rates are consistent with other studies investigating burnout in internal medicine residents (Ishak et al). ${ }^{5}$ 
Table I Prevalence of burnout at three time points

\begin{tabular}{|c|c|c|c|c|c|c|}
\hline \multirow[t]{2}{*}{ Burnout } & \multicolumn{2}{|c|}{$\begin{array}{l}\text { Time point I } \\
(\mathbf{n}=\mathbf{3 2})\end{array}$} & \multicolumn{2}{|c|}{$\begin{array}{l}\text { Time point } 2 \\
(n=3 I)\end{array}$} & \multicolumn{2}{|c|}{$\begin{array}{l}\text { Time point } 3 \\
(\mathrm{n}=\mathbf{2 4 )}\end{array}$} \\
\hline & $M / n$ & SD/\% & $M / n$ & SD/\% & $M / n$ & SD/\% \\
\hline & 21.91 & 7.95 & 21.32 & 8.20 & 17.33 & 8.34 \\
\hline MBI DP & 10.78 & 6.31 & 9.84 & 5.76 & 9.33 & 5.92 \\
\hline Total meeting criteria & 19 & $50 \%$ & 18 & $47.4 \%$ & 12 & $31.6 \%$ \\
\hline
\end{tabular}

Notes: Scores on the MBI EE $\geq 27$ are considered high burnout. Scores on the MBI $\mathrm{DP} \geq 10$ are considered high burnout.

Abbreviations: MBI, Maslach Burnout Inventory-Human Services; EE, emotional exhaustion subscale; DP, depersonalization subscale; $M$, mean; SD, standard deviation.

\section{Dispositional mindfulness as a predictor of burnout criteria}

A hierarchical logistic regression was performed to test whether dispositional mindfulness predicted burnout criteria. Gender, baseline stress, and baseline depression were entered at Step 1. The five subscales of the FFMQ were entered at Step 2 (Table 2). Results from the Homer and Lemeshow goodness-of-fit test compare the observed values with a perfect model and the test was non-significant, suggesting goodness of fit; $\chi^{2}(8)=6.59, p=0.58$. The second model significantly distinguished between residents who met criteria and those who did not meet criteria for burnout, $\chi^{2}(5)=11.88$, $p=0.04$. The model effect size was strong: Nagelkerke $\mathbf{R}^{2}=$ 0.64 . The model accurately predicted residents not meeting criteria for burnout $76.9 \%$ of the time, and was $89.5 \%$ accurate at predicting residents who did meet criteria for burnout, for an overall accuracy of $84.4 \%$. The "acting with awareness" subscale of the FFMQ was the only significant predictor of burnout criteria, with lower dispositional mindfulness increasing the likelihood of meeting criteria for burnout.

\section{Association between burnout and performance evaluations}

If residents met criteria for burnout at any one of the three time points, they were considered to have at least one episode of burnout during the study. One of the "patient care" performance milestones, "Manages patients with progressive responsibility and independence (PC3)" was significantly related to burnout, $\chi^{2}(1)=4.65, p=0.031$, such that meeting criteria for burnout was related to below average performance. Two other milestones, one measuring attendance at conferences and completion of paperwork, "Accepts responsibility and follows through on tasks (PROF2)", $\chi^{2}$ (1) $=4.40, p=0.036$, and another measuring patient referrals "Transitions patients effectively within and across health delivery systems (SBP4)", $\chi^{2}(1)=4.77, p=0.029$ were unexpectedly positively related to burnout; that is, meeting criteria for burnout was related to above average performance. Tables 3-5 present data from the $\chi^{2}$ classification tables for each milestone.

\section{Discussion}

This was the first study to show that dispositional mindfulness predicts burnout criteria. We also provided exploratory data on the effect of burnout on the Internal Medicine resident milestones developed by the ACGME. Burnout rates were high in this sample, providing evidence to support the need for efforts to improve burnout in internal medicine residents. However, this must be qualified given the low response rate. The hypothesis that dispositional mindfulness would predict burnout criteria was supported; specifically, "acting with awareness", the ability to attend to the moment, or the opposite of automatic pilot, may be especially helpful in reducing and/or protecting against burnout in internal medicine residents. Interventions developed to improve mindfulness may improve residents' resiliency and decrease burnout.

Exploratory analyses revealed that meeting criteria for burnout increased the likelihood of performing below average on the milestone "Manages patients with progressive responsibility and independence". Burnout was associated with a decrease in residents' ability to act independently as physicians - perhaps relying more on their attending or senior resident to make decisions.

Table 2 Hierarchical logistic regression of dispositional mindfulness predicting burnout

\begin{tabular}{lllllll}
\hline Step & Predictor & B & SE B & p-value & Exp(B) & 95\% CI \\
\hline Step I & Gender & -2.02 & 1.85 & 0.28 & 0.13 & $0.003-5.004$ \\
& Stress & 0.191 & 0.29 & 0.50 & 1.21 & $0.69-2.12$ \\
& Depression & -0.76 & 0.44 & 0.08 & 0.47 & $0.20-1.10$ \\
Step 2 & FFMQ Nonreact & -0.07 & 0.20 & 0.74 & 0.93 & $0.63-1.39$ \\
& FFMQ Observe & -0.29 & 0.21 & 0.17 & 0.75 & $0.50-1.13$ \\
& FFMQ ActAware & -0.59 & 0.29 & $0.04 *$ & 0.55 & $0.32-0.97$ \\
& FFMQ Describe & 0.05 & 0.23 & 0.84 & 1.05 & $0.67-1.63$ \\
& FFMQ Nonjudge & -0.11 & 0.17 & 0.51 & 0.89 & $0.64-1.25$ \\
\hline
\end{tabular}

Notes: ${ }^{*} p<0.05$. Nonreact: nonreactivity to inner experience. ActAware: acting with awareness. Nonjudge: nonjudging of inner experience.

Abbreviations: B, unstandardized beta weight; SE, standard error; Exp(B), odds ratio; Cl, confidence interval; FFMQ, Five Facet Mindfulness Questionnaire. 
Table 3 Chi-square classification table for milestone: "Manages patients with progressive responsibility and independence (PC3)"

\begin{tabular}{lllll}
\hline Milestone & Performance score & \multicolumn{2}{c}{ Burnout criteria } & \\
\cline { 3 - 4 } & & Not met & Met & Total \\
\hline PC3 Criteria & Below average & 2 & 12 & 14 \\
& At or above average & $1 \mathrm{I}$ & 12 & 23 \\
& Total & 13 & 24 & 37 \\
\hline
\end{tabular}

Abbreviation: PC, patient care.

Table 4 Chi-square classification table for milestone: "Transitions patients effectively within and across health delivery systems (SBP4)"

\begin{tabular}{lllll}
\hline Milestone & Performance score & \multicolumn{2}{c}{ Burnout criteria } & \\
\cline { 3 - 4 } & & Not met & Met & Total \\
\hline SBP4 Criteria & Below average & 7 & 4 & II \\
& At or above average & 6 & 18 & 24 \\
& Total & 13 & 22 & 35 \\
\hline
\end{tabular}

Abbreviation: SBP, system-based practice.

Table 5 Chi-square classification table for milestone: "Accepts responsibility and follows through on tasks (PROF2)"

\begin{tabular}{lllll}
\hline Milestone & Performance score & \multicolumn{2}{c}{ Burnout criteria } & \\
\cline { 3 - 4 } & & Not met & Met & Total \\
\hline PROF2 Criteria & Below average & 10 & 10 & 20 \\
& At or above average & 3 & 14 & 17 \\
& Total & 13 & 24 & 37 \\
\hline
\end{tabular}

Abbreviation: PROF, professionalism.

Surprisingly, the milestones "Transitions patients effectively within and across health delivery systems" from the "system-based practice" competency and "Accepts responsibility and follows through on tasks" from the "professionalism" competency were related to burnout criteria such that meeting criteria for burnout increased the likelihood of performing at or above average. Discussion with program directors revealed that these milestones assessed attendance at weekly conferences, completion of paperwork, and generally, higher levels of professional behavior. Striving to perform at a higher level of professional care may lend itself to higher burnout in residents at a cost to their self-care. However, more research is necessary to cross-validate this relationship in larger samples.

If these findings are replicated, it may suggest a need to discover individual- and system-level approaches that will decrease burnout without sacrificing professionalism and attendance-based performance. It should be recognized that burnout may be promoting certain competencies of residency at a cost to both resident health and other competencies of work-related performance, including patient care.

Study limitations include the small sample size, which limits the generalizability and interpretation of findings.
Further, it limited the power to detect statistical significance, especially in tests of the relationship between performance and burnout. Notably, adjusting for family-wise error rate using a Bonferroni correction made these analyses nonsignificant. Therefore, it is possible that the three significant associations between Internal Medicine resident milestones and burnout were simply due to multiple comparisons. However, these tests are exploratory in nature and should be qualified as such. These results should be used to inform future studies investigating the relationship between Internal Medicine resident milestones and burnout. The sample size also limits the interpretation of the logistic regression investigating the relationship between dispositional mindfulness and burnout, as the sample size is smaller than recommended for this type of analysis. However, all assumptions were met and the model indicated a good fit; therefore, the relationship between burnout and dispositional mindfulness presented here warrants qualified consideration and future investigations to build upon these findings. This study is also limited by the static diagnosis of burnout caused by grouping residents as burnt out if they met criteria at any time point. However, residents who met criteria for burnout throughout the first half of a residency year may be different than residents who did not; therefore, these differences are especially compelling in how they relate to performance and what factors may be protective against burnout, including mindfulness.

The response rate in this study prevents elimination of selection bias as a potential confounding variable. Although it was developed by experts and has good face and content validity ${ }^{31}$ the measure of performance has not been validated against external criteria, given that this is the first study to investigate the relation between Internal Medicine resident milestones from program directors and burnout. Second year residents were underrepresented in this study due to difficulties in recruitment scheduling, thereby limiting generalizability. Finally, this study was limited by assessing only one internal medicine program, as well as by assessing only one specialty. However, these preliminary data may inform future larger scale studies. Subsequent research should evaluate several medical residency specialties, throughout their entire residency, on measures of burnout and investigate effects on resident performance evaluations.

\section{Conclusion}

Burnout may be associated with better performance on some competencies and poorer performance on other competencies; therefore, strategic intervention should be investigated 
to preserve work-related performance while improving resident health. Interventions that improve mindfulness may be especially helpful for decreasing burnout, as our findings suggest here, while also improving performance, as demonstrated elsewhere. ${ }^{38-41}$ More research is needed to understand the effects of burnout on performance in medical residents and how program directors across specialties may help to ameliorate these effects.

\section{Acknowledgments}

The authors wish to thank the Department of Internal Medicine for all their support and the residents themselves for providing their time, of which they have so little! This work was supported by the HRSA/Graduate Psychology Education grant under Grant D40HP25724; HRSA/Graduate Psychology Education grant under Grant D40HP19638, and the Virginia Health Care Foundation. This paper was presented as a poster at the annual meeting of Women's Health Research Day at VCU, March 2015, in Richmond, VA, USA and at the annual meeting of the Society for Behavioral Medicine, April 2016, in Washington, DC, USA.

\section{Disclosure}

The authors report no conflicts of interest in this work.

\section{References}

1. Maslach C, Jackson SE, Leiter MP. Maslach Burnout Inventory: Third edition. In: Zalaquett CP, Wood RJ, editors. Evaluating Stress: A Book of Resources. The Scarecrow Press; 1997:191-218.

2. Maslach C, Jackson S. The measurement of experienced burnout. Journal of Organizational Behavior. 1981;2(2):99-113.

3. Thomas NK. Resident burnout. JAMA. 2004;292(23):2880-2889.

4. Shanafelt TD, Boone S, Tan L, et al. Burnout and satisfaction with work-life balance among US physicians relative to the general US population. Arch Intern Med. 2012;172(18):1377-1385.

5. Ishak WW, Lederer S, Mandili C, et al. Burnout during residency training: a literature review. J Grad Med Educ. 2009;1(2):236-242.

6. Garman AN, Corrigan PW, Morris S. Staff burnout and patient satisfaction: evidence of relationships at the care unit level. J Occup Health Psychol. 2002;7(3):235-241.

7. Shanafelt TD, Bradley KA, Wipf JE, Back AL. Burnout and self-reported patient care in an internal medicine residency program. Ann Intern Med. 2002;136(5):358.

8. Prins JT, van der Heijden FM, Hoekstra-Weebers JE, Bakker AB, van de Wiel HB, Jacobs B, Gazendam-Donofrio SM. Burnout, engagement and resident physicians' self-reported errors. Psychol Health Med. 2009;14(6):654-666.

9. West CP, Tan AD, Habermann TM, Sloan JA, Shanafelt TD. Association of resident fatigue and distress with perceived medical errors. JAMA. 2009;302(12):1294-1300.

10. Halbesleben JR, Rathert C. Linking physician burnout and patient outcomes: exploring the dyadic relationship between physicians and patients. Health Care Manage Rev. 2014;33(1):29-39.

11. Lu DW, Dresden S, McCloskey C, Branzetti J, Gisondi MA. Impact of burnout on self-reported patient care among emergency physicians. West J Emerg Med. 2015;16(7):996-1001.
12. West CP, Huschka MM, Novotny PJ, et al. Association of perceived medical errors with resident distress and empathy: a prospective longitudinal study. JAMA. 2006;296(9):1071-1078.

13. West CP, Shanafelt TD, Kolars JC. Quality of life, burnout, educational debt, and medical knowledge among internal medicine residents. JAMA. 2011;306(9):952-960.

14. Shanafelt TD, Balch CM, Bechamps G, et al. Burnout and medical errors among American surgeons. Ann Surg. 2010;251(6):995-1000.

15. Kabat-Zinn J. Full Catastrophe Living: Using the Wisdom of Your Body and Mind to Face Stress, Pain, and Illness. Bantam; 1990.

16. Lamothe M, Rondeau É, Malboeuf-Hurtubise C, Duval M, Sultan S. Outcomes of MBSR or MBSR-based interventions in health care providers: A systematic review with a focus on empathy and emotional competencies. Complement Ther Med. 2016;24:19-28.

17. Burton A, Burgess C, Dean S, Koutsopoulou GZ, Hugh-Jones S. How effective are mindfulness-based interventions for reducing stress among healthcare professionals? A systematic review and meta-analysis. Stress Health. 2016;33(1):3-13.

18. Creswell JD, Lindsay EK. How does mindfulness training affect health? A mindfulness stress buffering account. Current Directions in Psychological Science. 2014;23(6):401-407.

19. Epstein RM, Krasner MS. Physician resilience: what it means, why it matters, and how to promote it. Acad Med. 2013;88(3):301-303.

20. Quaglia JT, Braun SE, Freeman SP, McDaniel MA, Brown KW. Metaanalytic evidence for effects of mindfulness training on dimensions of self-reported dispositional mindfulness. Psychol Assess. 2016;28(7): 803-818.

21. Brown KW, Ryan RM. The benefits of being present: mindfulness and its role in psychological well-being. J Pers Soc Psychol. 2003;84(4): 822-848.

22. Baer RA, Smith GT, Lykins E, et al. Construct validity of the five facet mindfulness questionnaire in meditating and nonmeditating samples. Assessment. 2008;15(3):329-342.

23. Krasner MS, Epstein RM, Beckman H, Suchman AL, Chapman B, Mooney CJ, Quill TE. Association of an educational program in mindful communication with burnout, empathy, and attitudes among primary care physicians. JAMA. 2009;302(12):1284-1293.

24. Van Der Linden D, Keijsers GP, Eling P, Van Schaijk R. Work stress and attentional difficulties: An initial study on burnout and cognitive failures. Work \& Stress. 2005;19(1):23-36.

25. Deligkaris P, Panagopoulou E, Montgomery AJ, Masoura E. Job burnout and cognitive functioning: A systematic review. Work \& Stress. 2014;28(2):107-123.

26. Sokka L, Leinikka M, Korpela J, et al. Job burnout is associated with dysfunctions in brain mechanisms of voluntary and involuntary attention. Biol Psychol. 2016;117:56-66.

27. Rabatin J, Williams E, Baier Manwell L, Schwartz MD, Brown RL, Linzer M. Predictors and outcomes of burnout in primary care physicians. J Prim Care Community Health. 2016;7(1):41-43.

28. Fahrenkopf AM, Sectish TC, Barger LK, et al. Rates of medication errors among depressed and burnt out residents: prospective cohort study. BMJ. 2008;336(7642):488-491.

29. Toker S, Shirom A, Shapira I, Berliner S, Melamed S. The association between burnout, depression, anxiety, and inflammation biomarkers: C-reactive protein and fibrinogen in men and women. J Occup Health Psychol. 2005;10(4):344-362.

30. Melamed S, Shirom A, Toker S, Berliner S, Shapira I. Burnout and risk of cardiovascular disease: evidence, possible causal paths, and promising research directions. Psychol Bull. 2006;132(3):327-353.

31. Green ML, Aagaard EM, Caverzagie K, et al. Charting the road to competence: Developmental milestones for internal medicine residency training. J Grad Med Educ. 2009;1(1):5-20.

32. Accreditation Council for Graduate Medical Education. The Internal Medicine Milestone Project. ACGME \& ABIM; 2013. Available from: http://www.acgme.org/acgmeweb/tabid/134/ProgramandInstitutiona1Accreditation/MedicalSpecialties/InternalMedicine.aspx. Accessed April 1, 2017. 
33. Rafferty JP,Lemkau JP, Purdy RR, Rudisill JR. Validity of the Maslach Burnout Inventory for family practice physicians. J Clin Psychol. 1986;42(3): 488-492.

34. Landrigan CP, Fahrenkopf AM, Lewin D, et al. Effects of the accreditation council for graduate medical education duty hour limits on sleep, work hours, and safety. Pediatrics. 2008;122(2):250-258.

35. Bohlmeijer E, ten Klooster PM, Fledderus M, Veehof M, Baer R. Psychometric properties of the five facet mindfulness questionnaire in depressed adults and development of a short form. Assessment. 2011;18(3): 308-320.

36. Antony MM, Bieling PJ, Cox BJ, Enns MW, Swinson RP. Psychometric properties of the 42-item and 21-item versions of the Depression Anxiety Stress Scales in clinical groups and a community sample. Psychological Assessment. 1998;10(2):176-181.
37. Henry JD, Crawford JR. The short-form version of the Depression Anxiety Stress Scales (DASS-21): construct validity and normative data in a large non-clinical sample. Br J Clin Psychol. 2005;44(Pt 2):227-239.

38. Chambers R, Lo BC, Allen NB. The impact of intensive mindfulness training on attentional control, cognitive style, and affect. Cognit Ther Res. 2008;32(3):303-322.

39. Jha AP, Krompinger J, Baime MJ. Mindfulness training modifies subsystems of attention. Cogn Affect Behav Neurosci. 2007;7(2):109-119.

40. Zeidan F, Johnson SK, Diamond BJ, David Z, Goolkasian P. Mindfulness meditation improves cognition: evidence of brief mental training. Conscious Cogn. 2010;19(2):597-605.

41. Chiesa A, Calati R, Serretti A. Does mindfulness training improve cognitive abilities? A systematic review of neuropsychological findings. Clin Psychol Rev. 2011;31(3):449-464.
Advances in Medical Education and Practice

\section{Publish your work in this journal}

Advances in Medical Education and Practice is an international, peerreviewed, open access journal that aims to present and publish research on Medical Education covering medical, dental, nursing and allied health care professional education. The journal covers undergraduate education, postgraduate training and continuing medical education

\section{Dovepress}

including emerging trends and innovative models linking education, research, and health care services. The manuscript management system is completely online and includes a very quick and fair peer-review system. Visit http://www.dovepress.com/testimonials.php to read real quotes from published authors.

Submit your manuscript here: http://www.dovepress.com/advances-in-medical-education-and-practice-journal 